\title{
L'immigration haïtienne au Venezuela : identité et intégration
}

\section{Alain Charier}

\section{OpenEdition}

\section{Journals}

Édition électronique

URL : http://journals.openedition.org/plc/499

DOI : $10.4000 /$ plc.499

ISSN : 2117-5209

Éditeur

L'Harmattan

Édition imprimée

Date de publication : 1 janvier 1999

Pagination : 175-197

ISSN : 1279-8657

\section{Référence électronique}

Alain Charier, «L'immigration haïtienne au Venezuela : identité et intégration», Pouvoirs dans la Caraïbe [En ligne], 11 | 1999, mis en ligne le 14 mars 2011, consulté le 20 avril 2019. URL : http:// journals.openedition.org/plc/499; DOI : 10.4000/plc.499 


\section{L'immigration haïtienne au Venezuela : identité et intégration}

par Alain CHARIER

Membre associé du CRPLC

\section{L'IMMIGRATION HAITIENNE AU VENEZUELA : IDENTITE ET INTEGRATION}

Bien que l'immigration haïtienne au Venezuela ne soit pas l'une des plus importantes numériquement qu'ait connue ce pays, les souvenirs de liens historiques ambigus, les préjugés et les stéréotypes de tous ordres envers Haïti et sa population se sont conjugués pour rendre ce thème particulièrement sensible.

A cela s'ajoute le fait que l'on ne connaisse encore que relativement peu de choses sur les réalités de cette communauté (motivations, composition socio-démographique, voies d'insertion dans la société d'accueil) qui a beaucoup moins attiré l'attention des chercheurs que ses homologues d'Amérique du Nord ou de la Caraïbe insulaire. Perçue autrefois par l'opinion vénézuélienne comme étant une conséquence directe de la répression duvaliériste que la démocratie se devait donc, sinon de soutenir, du moins d'accepter, elle est désormais vécue comme un transfert chez elle de la misère et de l'anarchie endémiques qui règnent dans la république insulaire.

L'approche que nous présentons ici essaye d'explorer, à partir d'une expérience originale, les pistes qui pourraient être celles d'une recherche visant à la compréhension des logiques qui structurent cette communauté immigrée caractérisée par la complexité de son histoire, 
de ses liens avec le Venezuela et des déterminants qui conditionnent son insertion.

Les études réalisées au Venezuela sur les phénomènes migratoires se sont généralement limitées aux aspects quantitatifs de ceux-ci (avec souvent comme nous le verrons une marge d'imprécision importante) et se sont peu intéressées à leur impact socio-culturel contemporain ${ }^{1}$.

Nous avons cherché à prendre en compte, dans le cadre limité du présent article, le contexte politique et social général qui a orienté cette immigration vers le Venezuela ainsi que les motivations individuelles et collectives de celle-ci, les causes de sa concentration géographique, les facteurs qui rendent son intégration problématique, ses liens maintenus ou recréés avec Haïti et les autres diasporas, ses modes d'organisation anciens et récents, ses relations enfin avec la composante noire de sa société d'accueil.

\section{HISTORIQUE ET COMPOSITION DE LA COMMUNAUTE : LES DEUX VAGUES DE L'IMMIGRATION}

La communauté haïtienne résidant actuellement au Venezuela est majoritairement de formation récente. De 1959 à 1986, le pays a accueilli principalement, mais pas uniquement, des exilés politiques et leurs familles parfois élargies. Cette situation n'a rien d'original en Amérique Latine où s'étaient réfugiés par exemple des intellectuels tels que Gérard Pierre-Charles, Suzy Castor ou Rosny Smart.

Cette première vague ne se soucia pas de structurer la communauté, ni de faire connaître la réalité sociale et culturelle haïtienne auprès de la population vénézuélienne. Ses efforts étaient alors tendus vers la prise du pouvoir en Haïti et vers le retour à Port-

1. Berglund. S, Hernandez-Caliman. H., Los de afuera, un estudio analitico del proceso migratorio en Venezuela. 1936-1985, Caracas, CEPAM, 1985.

Torrealba R, Oropeza J. A., Estado y migraciones laborales en Venezuela, Ed. Cabildo, Caracas, 1988. 
au-Prince. La dictature se prolongeant, une nouvelle génération naquit et grandit dans l'exil ; elle chercha le plus souvent à s'intégrer dans la société d'accueil.

L'immigration plus récente se différencie de la précédente par son origine sociale modeste et sa composante rurale dominante, elle correspond à ce que les élites en Haïti appellent le «pays en dehors », titre d'un ouvrage de Gérard Barthelemy², qui a fait irruption sur la scène politique lors de l'arrivée au pouvoir du mouvement Lavalas.

La situation catastrophique des campagnes haïtiennes due à la déforestation, à la sécheresse, mais aussi à la concurrence déloyale des excédents agricoles américains est pour beaucoup dans cet exode. Echapper à la sous-alimentation, voire à la famine, et au manque de perspectives offertes par un Etat classé au $156^{\mathrm{e}}$ rang des nations par le PNUD et au dernier rang en Amérique sont les motivations essentielles de cette diaspora.

Dans un tel contexte le choix du Venezuela semble n'être qu'un choix par défaut imposé par la fermeture progressive des destinations naguère privilégiées : Etats-Unis, Canada, départements français de la Caraïbe. Caracas où l'administration est moins efficace, où des possibilités de corruption existent et pour laquelle des filières d'accès se sont constituées à Port-au-Prince, reste beaucoup plus accessible. Sur ce point Salvador Prevau, Secrétaire général de la «Comunidad de Haïti au Venezuela» (Cohave) déclare : «Les Haïtiens arrivent souvent au Venezuela parce qu'ils ont été trompés, dans l'île agissent des gens qui organisent les entrées illégales, ils ont des contacts avec des employés de l'aéroport de Maiquetia $»^{3}$. Certes le Venezuela est en crise et l'âge d'or des années 70 qui avait favorisé une importante immigration latino-américaine et caraibéenne n'est plus qu'un lointain

2. Barthelemy G., Le pays en dehors, Port-au-Prince, Ed. Henri Deschamp, 1987.

3. Chiappe D., «El drama de los inmigrantes haitianos, llegan engañados y regresan escarmentados », El Nacional, Caracas, 21 février 1999. 
souvenir mais, pour le paysan haïtien, toute opportunité mérite plus que jamais d'être saisie ${ }^{4}$.

Pendant longtemps les immigrants se sont consacrés principalement à la vente de vêtements, d'abord à la sauvette («buhoneros ») ou sur les marchés, puis certains qui avaient prospéré ouvrirent leurs propres magasins dans les quartiers populaires de la capitale ou de certaines grandes villes de province. Un réseau d'approvisionnement reliant ces commerçants à la zone franche de Margarita s'est même organisé. Il s'agissait généralement de membres de la première vague, qui possédaient un certain niveau d'instruction et étaient par là même capables de mener à bien des entreprises commerciales complexes nécessitant une certaine maîtrise de l'espagnol.

A partir de 1986 environ on note une certaine réorientation des activités de la communauté haïtienne, les nouveaux venus se dirigeant désormais vers la vente ambulante de glaces. Les raisons d'un tel « choix » sont multiples : il s'agit d'une activité plus aisée à pratiquer pour des personnes non hispanophones, mais surtout il existe à Caracas un réseau de recrutement à base parentale ou géographique qui permet à ces immigrants, majoritairement illégaux, de trouver de suite un travail. Les grandes compagnies de distribution ont un intérêt bien compris à assurer la pérennité de ce système, car elles savent que ces Haïtiens ruraux restent redevables envers leurs parents ${ }^{5}$ qui leur ont permis l'accès à cet emploi et seront fiables et dépendants du bon vouloir de l'entreprise. La silhouette de l'Haïtien vendeur de glace s'est peu à peu imposée dans le paysage quotidien de la capitale, au point que le photographe Guillermo Suarez - récompensé pour ce

4. «Se hunde barco con hasta 18 haïtiano en la costa de Florida », El Nuevo Heraldo, Miami, 7 mars 1999. L'article souligne l'augmentation du nombre des boatpeoples haïtiens repêchés au large de la Floride durant les deux dernières années, ceuxci passant de 587 en 1997 à 1206 en 1998.

Cassen B., « Haïti dans la spirale du désespoir », Le Monde Diplomatique, Octobre 1997.

Hurbon L. «Haïti entre la guerre froide et le nouvel ordre mondial », in Les Transitions Démocratiques, Paris, Syros, 1996.

5. Bastien R., Le paysan haïtien et sa famille, Paris, Karthala, 1985. 
travail par le jury du concours « Banco de imagenes 1997 » leur a consacré une exposition intitulée »Venezuela : Désespoir ou solution pour les Haïtiens ?» et ainsi dédicacée : «Vendiendo helados de cualquier marca o sabor... en nuestro comun trajinar, con sus costumbres, su historia, su lengua y sus anhelos $»^{6}$.

\section{Une présence difficilement quantifiable}

Evaluer l'importance démographique de la communauté haïtienne vivant au Venezuela s'avère être une tâche particulièrement complexe. Tout d'abord, l'immense majorité des membres de la première vague d'immigration ont désormais acquis la nationalité vénézuélienne et figurent donc comme tels dans les recensements (rappelons qu'une loi de 1926 interdit de faire état à cette occasion de la couleur de peau ou des origines ethniques); il en est de même de la génération née sur place (la double nationalité reste impossible). Au sein de la nouvelle immigration majoritairement illégale, voire clandestine, il n'y a pas de contacts avec les autorités locales et peu avec l'ambassade. Même pour les Haïtiens entrés légalement le chemin qui mène à la naturalisation est long et difficile, la DIEX (administration qui gère les visas et les permis de séjour) les maintient le plus longtemps possible sous le régime dit de «la boleta » qui les oblige à se présenter périodiquement dans l'espoir d'une régularisation, parfois durant des années, les faisant tomber ainsi finalement dans l'illégalité alors que certains ont déjà des enfants qui sont eux vénézuéliens de naissance ${ }^{7}$. Les chiffres qui sont cités ici doivent donc être considérés avec beaucoup de prudence.

Une enquête diligentée par les autorités haïtiennes en 1986 faisait état d'environ 25000 résidents, selon les mêmes sources 7000 sont présents aujourd'hui (dont 800 soumis au système de «la boleta $\gg)^{7}$. Ce déclin s'expliquant à la fois par quelques retours au

6. «Tres proyectos que desatan el sentimiento de desarraigo », El Globo, Caracas, 8 juillet 1997.

7. Article de El Nacional déjà cité. 
pays, par les départs vers les Etats-Unis et le Canada et par les naturalisations. Ces données semblent être très en deçà de la réalité ; il en est de même en ce qui concerne celles qui émanent des instituts vénézuéliens, ces dernières ne manquent pas cependant d'être instructives.

Selon le recensement de $1990^{8}$, dernier en date, on dénombrait seulement 1796 haïtiens résidant au Venezuela. Il faut noter que l'on se trouve alors à la veille de l'arrivée au pouvoir de J. B. Aristide. Cette communauté est à $65 \%$ masculine (1 018 hommes, 778 femmes) et géographiquement regroupée : on recense en effet 1432 haitiens dans la région de Caracas. La seconde concentration se situe dans l'état de Lara (102) et plus précisément à Barquisimeto où un marché libre a donné une relative stabilité économique à la communauté implantée localement. Toujours selon le même recensement on ne comptait que 262 Haïtiens disséminés sur tout le reste du territoire - certains états n'en comptant qu'un seul, deux groupes un peu plus importants sont cependant identifiables à Maracay et à Valencia. Etant surtout commerçants, ces immigrants sont essentiellement urbains. On est donc en présence d'un double mouvement migratoire : Haïti/Venezuela et campagne/ville; il s'agit donc bien là d'un processus fort différent de celui observé par exemple en République Dominicaine où les Haïtiens - dont le nombre est évalué à 500000 - se consacrent à la coupe de la canne ou dans les DOM où ils sont également employés avant tout dans le secteur agricole?

Les données les plus récentes dont on peut disposer remontent à 1995 et sont relatives aux entrées et sorties du territoire ${ }^{10}$. A cette date J. B. Aristide est revenu au pouvoir (19 septembre 1994) mais la crise économique a continué de s'aggraver. Cette année là les sorties (839) sont supérieures en nombre aux entrées (684). L'observation de la répartition des types de visa se révèle particulièrement instructive : 48

8. El censo en Venezuela XII Censo general de poblacion y vivienda, OCEI, Caracas, pp. 47-61.

9. Hurbon L., «Démocratisation, identité culturelle et identité nationale », Pouvoirs dans la Caraïbe $\mathrm{n}^{\circ}$ 10, 1998, p. 219.

10. Anuario estadistico de Venezuela, 1995, OCEI, Caracas, 1996, p. 231. 
visas d'entrée comme «transeunte » (autant de sorties) c'est-à-dire d'autorisation de travail pour une période limitée, 314 de tourisme (432 sorties) ce qui ne peut manquer de surprendre, 239 de résidents (314 sorties) vivant de façon permanente dans le pays, 42 non spécifiés ! (41 sorties) et enfin 41 de transit ce qui est d'autant plus étrange que l'on ne compte que 4 sorties sous cette rubrique ${ }^{11}$.

Plusieurs constatations s'imposent sur la base de ces statistiques : on observe tout d'abord que seule une fraction réduite de la communauté serait mobile, on note ensuite que le visa de tourisme, facile à obtenir puis à contourner, est le plus utilisé. Enfin il apparaît que de toute évidence les mouvements migratoires au Venezuela font l'objet d'un contrôle sporadique et partiel. Comme le résumait, pour le déplorer, un journaliste à propos précisément de la présence haïtienne : «El asunto es que en Venezuela no parece haber control de inmigrantes y eso no puede ser $»^{12}$. Il renvoyait ainsi de fait aux complicités locales évoquées précédemment.

Les immigrants récents sont dans leur majorité des jeunes de 25 à 35 ans ; à Caracas cette deuxième vague migratoire vit surtout dans le quartier de Los Magallanes à Catia (vaste banlieue populaire de l'ouest de la capitale). Les nouveaux venus s'installent d'abord chez un parent, puis ils cherchent un logement situé à proximité et l'aire occupée par les Haïtiens s'étend ainsi peu à peu. On peut observer d'autres regroupements de ce type, plus modestes, dans l'Est de l'agglomération, à Guarenas par exemple.

\section{HAÏTI-VENEZUELA : UNE RELATION COMPLEXE}

Le peuple haïtien perçoit en général les nations latinoaméricaines hispanophones comme des pays «blancs » ou du moins

11. Les activités de propriétaires de navires ancrés dans le port de La Guaïra et qui emploient des Haïtiens à leurs bords pour lesquels ils obtiennent des visas de transit avant de les introduire au Venezuela de manière définitive ont été récemment dénoncées. Article de El Nacional déjà cité.

12. Article de El Globo déjà cité. 
dirigés par des blancs et relativement hostiles à son égard. Les rapports orageux avec le voisin dominicain sont pour beaucoup dans la persistance de ce sentiment. L'invasion de Saint-Domingue par l'armée haïtienne au siècle dernier, les massacres des travailleurs haïtiens perpétrés par la dictature de L. Trujillo, les échanges de bons procédés entre ce dernier et $\mathrm{F}$. Duvalier effectués au détriment des coupeurs de canne ${ }^{13}$, l'aide discrètement apportée aux militaires lors de leur putsch contre J. B. Aristide puis lors de leur fuite, le racisme de la dernière campagne présidentielle dominicaine qui a vu s'agiter une fois de plus le spectre du «péril haïtien », les profits retirés de l'embargo des années 1991-1994 par l'Etat hispanophone semblent avoir creusé un fossé infranchissable entre les deux communautés. Au Venezuela celles-ci demeurent séparées et au mieux indifférentes l'une à l'autre.

Les Haïtiens sont conscients de leur grande infériorité numérique (selon le recensement de 1990 on comptait neuf fois plus de ressortissants dominicains au Venezuela) mais surtout de leur plus grand éloignement culturel de la société d'accueil ${ }^{14}$. L'intégration des dominicains n'est cependant pas non plus sans problèmes, car si elle se trouve facilitée notamment par l'usage de la même langue et par un phénotype en général plus proche, ceux-ci restent cantonnés dans les secteurs informels de l'économie et habitent également les quartiers périphériques et défavorisés des grandes villes ${ }^{15}$. L'importance du contraste qui existerait entre la situation des deux communautés est donc plus souvent fantasmée que réelle ; elle n'en demeure pas moins significative de la relation qu'entretiennent nombre d'haïtiens avec la société vénézuélienne. L'attitude de cette dernière vis à vis d'Haïti et des Haïtiens se caractérise quant à elle par un double langage : on insiste certes rituellement sur la fraternité d'armes passée en faveur de

13. Plant R., Sugar and modern slavery. A tale of two countries, London, Zed Books, 1987.

14. Entretiens avec Fritz Saint-Louis Président de l'association Maison de la Culture Haïtienne au Venezuela et avec d'autres membres de l'association, Caracas, 1997/1998.

15. Entretien avec Jesus Garcia Président de la fondation «Afroamérica» qui a mis en place un projet de recherche sur cette communauté en coopération avec certains de ses représentants. 
la liberté et de l'indépendance à l'époque où Pétion apportait son soutien à un Bolívar défait et exilé. Et il est vrai que parfois cette dette historique fut évoquée dans un passé récent lorsque par exemple le gouvernement du Président L. Herrera-Campins (1978-1983) favorisa la régularisation des immigrés haïtiens où quand des voix s'élevèrent pour soutenir l'accueil de 150 réfugiés se trouvant sur la base de Guantanamo à Cuba après avoir fui la dictature du Général R. Cedras. Durant cette période le Venezuela comptait avec la France, les EtatsUnis, le Canada puis l'Argentine au nombre des «Pays amis du Secrétaire général sur Haïti ». Cette solidarité trouva cependant vite ses limites dès lors que la démocratie était formellement rétablie à Port-auPrince comme l'ont fort bien montré les expulsions qui ont eu lieu récemment : le 15 janvier 1999, 97 ressortissants haïtiens - hommes et femmes, adultes et enfants - qui venaient à la DIEX renouveler leur demande de séjour sont détenus sur place sur décision conjointe de cette administration et du maire du municipe Libertador (centre de Caracas) José Ledezma (membre du parti Action Démocratique), transférés à Maracay et sans examen des cas particuliers - comme le demandait la Cohave - et avec une certaine brutalité embarqués sur un vol militaire à destination d'Haïti. A cette occasion le Vice-président de Cohave déclara «Personne n'a l'intention de se réfugier dans un pays s'il pense qu'il y sera humilié et encore moins au Venezuela où il a existé historiquement une solidarité mutuelle avec Haïti... nous pensons qu'il y a eu discrimination raciale ${ }^{16}$. Soulignons au passage que le sort réservé aux cubains se trouvant dans la même situation a toujours été fort différent. Il est vrai que ceux-ci disposent de relais politiques au Venezuela. Comme l'a souligné à juste titre A. CastilloLevison dont nous reprenons ici les conclusions: «La population haïtienne... se situe dans le conflit inter-ethnique, quand la population d'accueil vit mal la présence du nouveau type d'immigrant et applique par extrapolation à l'Haïtien une série de préjugés portés sur d'autres groupes par le passé, auxquels vont s'ajouter des préjugés raciaux. On comprend alors la difficulté d'insertion et d'intégration de l'Haïtien dans une société urbaine problématique où les relations entre les individus sont marquées par les origines ethniques et les différences

16. Article de El Nacional déjà cité. 
socio-économiques. Ces différences se manifestent de façon permanente dans le discours et les relations quotidiennes que les Vénézuéliens entretiennent avec leurs concitoyens noirs et les groupes immigrants ethniquement différenciés tels les Haïtiens. Ces caractéristiques socioculturelles et ethniques semblent les conduire à la non-intégration dans la société d'accueil ${ }^{17}$.

\section{Des regards divergents sur un même processus migratoire}

Selon Béatrice Pouligny-Morgant : «La présence d'un nombre important d'Haïtiens à l'extérieur constitue une donnée fondamentale de ce rapport à l'autre, à la fois mythe du départ... et porte ouverte sur l'extérieur, sur la modernité, au sens sociologique du terme ${ }^{18}$. Par contre l'accueil qui est trop souvent réservé à ces immigrants au Venezuela s'ancre quant à lui dans le rêve toujours frustré de blanchir le pays grâce à une immigration sélective.

$\mathrm{Au}$ lendemain de l'indépendance, le groupe créole qui n'entendait pas voir une immigration noire contrarier ses projets de développement tenta de favoriser l'immigration européenne (loi de 1823). Durant la seconde moitié du dix-neuvième siècle et le début du vingtième des textes successifs tentèrent - sans succès - d'interdire toute arrivée de population en provenance de la Caraïbe insulaire. A cette époque le Venezuela connut l'essor d'un puissant courant positiviste (G. Fortoul, L. Vallenilla-Lanz), très écouté du pouvoir, qui soutenait la thèse selon laquelle seul un Venezuela «blanchi »serait à même d'entrer dans la modernité.

Ce n'est que durant la période de la politique migratoire dite de la «porte ouverte»(1948-1958) que cet objectif sera précairement atteint : c'est ainsi qu'en 1950 sur 208731 résidents nés à l'extérieur

17. Castillo-Levison A., La migration hä̈tienne au Venezuela. Un cas d'étude de l'insertion à l'intégration, Paris III, 1987, non publié, résumé.

18. Pouligny-Morgant B., «L'intervention de l'ONU dans l'histoire politique récente d'Haïti les effets paradoxaux d'une interaction », Pouvoirs dans la Caraïbe $\mathrm{n}^{\circ} 10$, p. 180 . 
127000 sont européens (essentiellement italiens, espagnols et portugais) et qu'en 1961 le rapport passe à 329850 sur un total de $541563^{19}$. Cette immigration blanche massive va cristalliser un peu plus les oppositions raciales car ces Européens vont avoir tendance à se regrouper entre eux notamment au sein d'associations et de clubs dont l'accès est fermé aux Vénézuéliens de couleur ${ }^{20}$ vis-à-vis desquels ils conservent une certaine réticence notamment du fait de l'absence de population noire dans leurs pays d'origine respectifs à cette époque. De plus les facilités - relatives - d'installation qui leur ont été accordées vont créer une amertume au sein des groupes noirs et mulâtres qui vont par la suite attribuer, à tort, à ces nouveaux arrivants l'origine du racisme latent dont ils se sentent victimes. Cette situation sera d'ailleurs habilement utilisée par les élites locales, qui bien que multipliant les alliances économiques et sociales avec ces immigrants, sauront détourner sur eux les rancœurs des couches défavorisées de la population.

Ce blanchiment est cependant radicalement remis en cause avec l'avènement de la période dite de la « Venezuela saudita » (1973-1983) qui voit l'apparente richesse du pays au lendemain de la première crise pétrolière attirer une immigration latino-américaine et caribéenne massive, alors que dès 1974-1975 la composante européenne devient marginale ${ }^{20}$. Les chiffres du recensement de 1990 rendent compte de ce bouleversement : sur 1025894 résidents nés à l'étranger 686716 sont latino-américains (508 166 colombiens) ou caribéens alors que 255899 seulement sont européens ${ }^{20}$.

Or c'est justement dans ce contexte que va se produire la seconde vague de l'immigration haïtienne alors que les groupes dirigeants voient s'éloigner, semble-t-il définitivement, leur rêve séculaire et que l'aggravation constante de la crise sociale et économique rend impopulaire au sein des couches défavorisées l'arrivée de concurrents potentiels prêts à accepter n'importe quelle condition de travail car n'ayant rien à perdre. Dès lors le sentiment

19. Bolivar Chollett M., Poblacion y sociedad en la Venezuela del siglo XX, Ed. Caracas, Tropykos, 1994, pp. 218-233.

20. Discrimination dont nous avons pu observer la persistance de nos jours. 
anti-haïtien latent va pouvoir se donner libre cours notamment dans les médias.

L'exil temporaire de J. B. Aristide à Caracas à l'invitation du Président C. A. Pérez donnera l'opportunité à une certaine presse - qui certes visait au-delà du président haïtien le chef d'état vénézuélien d'utiliser les stéréotypes racistes les plus éculés. Outre l'aimable sobriquet de «tache noire » (terme utilisé au Venezuela pour parler de la dégradation du revêtement de certaines routes) dont fut affublé J. B. Aristide, on prétendit « humoristiquement» que lui et le maire noir de Caracas de l'époque A. Isturiz ${ }^{21}$ n'étaient en fait qu'une seule et même personne laide et incompétente ; enfin et surtout on accusa le chef d'Etat déchu de vivre aux crochets du Venezuela alors qu'en réalité c'était l'ONU qui réglait ses frais de séjour. On voit apparaître ici en filigrane deux stéréotypes liés à Haïti : il s'agirait d'une part d'un agent déstabilisateur pour la société vénézuélienne (on pourrait faire remonter l'origine de ce sentiment à l'insurrection de Chirinos à Coro en 1795 derrière laquelle le pouvoir espagnol et les élites créoles virent l'influence des événements qui se déroulaient alors à Port-au-Prince) et d'autre part on serait en présence d'une nation perpétuellement assistée et cherchant constamment à parasiter ses voisins. Il faut souligner que «l'épisode Aristide » reste très présent au Venezuela, ainsi l'année dernière une allusion faite par le Président R. Préval à celui-ci dans l'un de ses discours l'obligea à annuler son escale à Caracas à la grande déception d'une communauté haïtienne qui se sentit, une fois de plus, oubliée.

21. Maire de Caracas de 1992 à 1995, membre du parti Causa R, il eut à souffrir de nombreuses attaques de la part d'une certaine presse à laquelle il n'hésita pas à répondre. 


\section{Le lien avec les autres diasporas : de la solidarité à la marginalisation}

De nombreux haïtiens résidant au Venezuela ont aussi de la famille aux Etats-Unis et au Canada ${ }^{22}$ et l'image de réussite sociale réelle ou supposée - dans des pays développés et plein d'avenir pour les immigrants que transmettent ces parents jointe, dans la période récente, à la détérioration de la situation économique et sociale ont conduit nombre de ceux qui en avaient la possibilité à rejoindre ces pays modifiant ainsi le solde migratoire.

Un resserrement des liens entre les communautés de Caracas, Boston, Miami et Montréal avait pu être observé durant la période de résistance à la dictature entre 1991 et $1994^{23}$. Cet échange était l'occasion pour des organisations œuvrant dans des cadres nationaux économiquement, socialement et culturellement différenciés de se connaître et de comparer leurs méthodes d'action. Il faut également rappeler qu'après son arrivée au pouvoir, le président J. B. Aristide avait créé un ministère dit du dixième département (le territoire de la République d'Haïti étant divisé en neuf entités de ce type) responsable des relations avec l'ensemble des communautés vivant à l'étranger. Dans le gouvernement de M. R. Smarth, c'était monsieur Paul Dejean qui occupait le poste de ministre des haïtiens de l'étranger. Cette prise en compte apparaissait comme logique de la part du gouvernement de Port-au-Prince, car la diaspora joue un rôle fondamental dans l'économie de l'île : elle est une source importante de devises ${ }^{24}$ pour un pays qui en manque cruellement. Certes l'apport de la petite

22. Il est bien évidemment impossible de savoir combien d'Haïtiens immigrés au Venezuela ont également des parents au Canada et aux Etats-Unis, mais ce thème revient de façon récurrente dans leur conversation souvent pour souligner que ces derniers ont fait un meilleur choix que le leur. Rappelons que la communauté nordaméricaine est évaluée à environ un million d'individus. L. Hurbon, article déjà cité, p. 219.

23. Entretien avec F. Saint-Louis qui a participé lui-même à ce processus.

24. On estime qu'elle envoie annuellement 800 millions de dollars pour une population d'un million d'exilés. Lionet Christian, «Haïti comme un vaisseau fantôme », Libération, Paris, 3 mars 1998. 
communauté du Venezuela rémunérée en bolívars, sans cesse dévalués, demeure sans aucun doute beaucoup plus modeste ${ }^{25}$ que celui de ses grandes sœurs nord-américaines et ce sont donc logiquement ces dernières qui retiennent plus l'attention des autorités centrales. Il existe par conséquent un sentiment diffus, mais réel, d'abandon par leur gouvernement de la part des haïtiens de Caracas.

Pour autant cherchent-ils à s'intégrer à la vie politique vénézuélienne ? En fait rien n'est moins sûr et on pourrait avancer plusieurs explications à ce phénomène : manque d'identification avec la société d'accueil et désir de rester à l'écart des débats qui l'agitent, espoir secret (mais sans doute de plus en plus ténu) de repartir un jour, analphabétisme, manque de culture démocratique, conscience d'une faiblesse numérique qui ne permet pas de peser sur les grandes orientations, absence d'un discours adapté de la part des hommes politiques. Cette liste n'est pas exhaustive, soulignons au passage que les Haïtiens ont tout loisir d'observer la remise en cause du système partisan en cours au Venezuela depuis une quinzaine d'années ${ }^{26}$ et la montée vertigineuse de l'abstention qui l'a accompagnée du moins jusqu'à l'élection de H. Chavez. Dans la période de toute puissance des appareils politiques (1958-1978), certains membres de la première vague d'immigration avaient su s'assurer une place au sein des diverses structures de pouvoir et ce au prix d'une identification plus complète à leur patrie d'accueil. Ces cas sont restés cependant isolés.

\section{AUX ORIGINES DU CHOIX DE L'ASSOCIATION «MAISON DE LA CULTURE HAÏTIENNE AU VENEZUELA »}

Notre approche de cette organisation doit s'inscrire dans la perspective d'une recherche sur le mouvement noir au Venezuela dont

25. Il serait aventureux de vouloir estimer le montant de l'apport de la communauté immigrée au Venezuela à l'économie haïtienne, mais au regard de son importance démographique et du Niveau de vie actuel de la société d'accueil il est évident qu'il reste marginal par rapport à Celui des diasporas nord-américaines.

26. Salamanca L., Crisis de la modernización y crisis de la democracia, Caracas, ILDIS, 1996. 
il nous était apparu nécessaire d'envisager la composante immigrée. Notre problématique générale se référait à l'émergence d'un Noir Sujet qui serait en mesure de revendiquer un rôle à l'intérieur des rapports sociaux et de contribuer ainsi à la construction d'une nouvelle modernité démocratique ${ }^{27}$, d'un Afro-vénézuélien capable d'intégrer une tradition revisitée à une (re)composition identitaire en devenir ; ce processus nécessitant pour sa concrétisation l'existence d'organisations noires structurées et porteuses d'objectifs clairs, engagées dans une action à la fois concrète et dynamique impliquant des partenaires multiples tant associatifs qu'officiels. Se posait dès lors le problème du choix des communautés à étudier, eu égard à la diversité des communautés noires qui cohabitent dans le Venezuela contemporain, cette diversité s'avérant être la conséquence de processus historiques anciens (esclavage) mais aussi - voire surtout - de facteurs socioéconomiques plus récents (urbanisation, immigration) dont l'impact sur la société toute entière n'a pu encore être pleinement évalué.

La communauté haïtienne qui est sans doute, nous l'avons vu, la plus en but aux préjugés concernant à la fois les noirs et les étrangers, celle qui rencontre probablement les plus grandes difficultés d'insertion et qui est la moins à même d'espérer une aide quelconque d'une patrie exsangue, mais qui est également la dépositaire d'une riche culture qui a su obtenir une reconnaissance au plan international (musique, peinture et littérature notamment au Québec avec E. Ollivier, S. Péan ou D. Laferrière) et d'une profonde tradition de solidarité (surtout s'agissant d'une immigration d'origine rurale) nous apparut comme particulièrement représentative de ces nouvelles identités noires en gestation. D'autant plus qu'elle évolue essentiellement dans un cadre urbain qui regroupe désormais $85 \%$ de la population vénézuélienne et dans lequel le renouveau noir joue désormais un rôle identitaire déterminant ${ }^{27}$.

La «Maison de la culture haïtienne », de par la conscience des enjeux que possèdent ses dirigeants, de par la stratégie d'intégration/ altérité qu'elle entend mettre en œuvre vis à vis de la société

27. Charier. A., «(Re)construcción de una identidad negra en Venezuela », Pouvoirs dans la Caraïbe $\mathrm{n}^{\circ} 10$, pp. 275-293. 
vénézuélienne et enfin de par sa collaboration effective avec des organisations noires autochtones nous est apparue comme étant une illustration, certes ponctuelle, mais prometteuse de ce que pourrait être l'avenir de communautés immigrées en profonde situation d'infériorité économique et sociale vis-à-vis de la société d'accueil mais capables de valoriser leur culture et aptes à nouer des alliances avec certains groupes de cette société avec lesquels des passerelles identitaires existent.

\section{Le désir de visibilité, la recherche de la cohésion}

En Haïti toute vie associative avait été détruite à l'époque de F. Duvalier ${ }^{28}$ et ce n'est qu'à partir de la chute du régime que les organisations nées de la société civile se manifestèrent, et ce dans tous les domaines. Elles acquirent même une telle importance qu'une féroce répression s'abattit sur elles au lendemain du coup d'état du Général R. Cedras ${ }^{29}$.

Certains fondateurs de l'association «Maison de la culture haïtienne au Venezuela» ont un passé fait de luttes politiques clandestines contre l'ancien régime et sont arrivés au Venezuela fuyant la police politique. C'est le cas de son président Fritz Saint-Louis qui a immigré en 1984 et est au demeurant membre de Amnesty international $^{30}$. D'autres membres ne se sont investis dans le combat politique que par la suite, tous enfin ont eu l'occasion de côtoyer à Caracas certains dirigeants en exil comme l'éphémère Président L. Manigat. Leur participation à la création de l'espace informatif en créole de la radio catholique «Fé y alegria » destiné à tenir au courant la communauté immigrée des événements se déroulant à Port-auPrince après la chute de J. B. Aristide joua également un rôle important

28. Etzen C., Le pouvoir politique en Haïti de 1957 à nos jours, ACCT, Paris, Karthala, 1994.

29. Jean-Claude J., «Société civile, promesses d'avenir », Bruxelles, Demain le Monde, Novembre 1997.

30. Sources sur historique et objectifs de l'association se reporter note 15 . 
dans leur engagement ultérieur. A cette époque une organisation de résistance à la dictature avait été fondée à Caracas, mais bientôt au sein même de celle-ci s'affrontèrent des thèses opposées: pour certains militants seul le combat visant au retour de la démocratie en Haïti valait la peine d'être mené, pour d'autres, au contraire, il fallait également profiter de cette mobilisation pour structurer une communauté qui dans son immense majorité ne reviendrait jamais au pays.

De telles circonstances permirent à ces militants de prendre conscience du peu d'intérêt réel des politiciens haïtiens pour leur diaspora dont la situation sociale et culturelle allait se détériorant alors que la société vénézuélienne continuait d'afficher une incompréhension totale à son égard. D'emblée leur souci fut donc de développer les possibilités de promotion de leurs concitoyens afin de les rendre « visibles » pour l'ensemble de la population et d'affirmer leur contribution potentielle au progrès de la nation tout entière. Les fondateurs de l'association se montrèrent extrêmement vigilants vis-àvis des hommes politiques pour éviter toute tentative de manipulation de leur part, ils choisirent d'établir un contact direct avec des membres de la communauté qu'ils avaient sélectionnés pour posséder un certain niveau d'instruction. Sur les cinquante personnes contactées, quarante répondirent à leur appel et participèrent à la mise sur pied du premier projet concret à savoir un cours d'enseignement de l'espagnol. Cette action fut à la base d'une nouvelle mobilisation s'appuyant sur la cérémonie de remise des diplômes linguistiques organisée à Parque Central le 30 décembre 1996. A partir de cette date, le noyau actif, désormais fort de 70 membres actifs, commença à se doter d'une réelle structure.

ORGANISATION ET STRATEGIE : PARTIR DES SOLIDARITES PREEXISTANTES

L'association est dirigée par un bureau exécutif de type classique et est divisée en trois sous-groupes : culture et information, relations publiques, formation. Elle cherche actuellement à se doter de cinq conseillers qui seraient choisis au sein des huit églises 
pentecôtistes haïtiennes existant au Venezuela qui sont les seuls lieux de rencontre pour les membres de la communauté.

Cette religion est en effet dominante au sein de la deuxième vague de l'immigration - rappelons qu'elle est en progression en Haïti même $\mathrm{e}^{31}$ - organisée en réseaux, favorisant les manifestations festives voire les phénomènes de transe, ne rompant pas ainsi avec la pensée magico-religieuse traditionnelle - elle dispose d'une grande capacité d'adaptation et d'une grande force de séduction. Si l'on ajoute aux caractéristiques que nous venons d'évoquer le fait que les cérémonies pentecôtistes soient célébrées dans la langue de la communauté en l'occurrence le créole (seuls $5 \%$ des haïtiens du Venezuela sont francophones) et non en espagnol comme celles de la religion catholique et que l'assemblée des fidèles est considérée comme une famille élargie ce qui favorise un sentiment de sécurité souvent absent au sein d'une petite population immigrée, on comprend d'autant mieux les raisons de son succès. Le rôle éducatif et social qu'elle assume n'est pas non plus à négliger, car celui-ci contribue à conforter des règles morales traditionnelles mises à mal par la confrontation avec une société d'accueil conflictuelle comme peut l'être la société vénézuélienne ; les lieux de culte offrent enfin un point de rencontre en l'absence d'un club haïtien comparable à ceux que possèdent au Venezuela les communautés immigrées plus nombreuses, plus riches et mieux organisées (italo-vénézuéliens, galiciens, syro-libanais...).

Le mouvement associatif naissant a donc logiquement décidé de mettre à profit cette capacité de rassemblement des églises et a délégué certains de ses membres auprès des responsables des diverses églises pentecôtistes haïtiennes. Au-delà de cette démarche, est apparue la nécessité pour l'organisation de disposer d'un espace propre de rencontre culturelle et sociale qui soit animé par des membres ayant reçu une formation spécialisée, cet espace pouvant jouer à terme un certain rôle économique en faveur de la communauté.

La «maison de la culture haïtienne au Venezuela » a également comme ambition de suppléer la présence défaillante de l'ambassade

31. Article de L. Hurbon déjà cité, p. 227. 
dans le domaine culturel, la section créée à cet effet au sein du personnel diplomatique sous la présidence de J. B. Aristide n'ayant jamais réellement fonctionné. L'unique solution en fait, compte tenu des contraintes matérielles des uns et des autres, semble résider dans une coopération la plus étroite possible entre le mouvement associatif et les organismes officiels. Ce principe est en passe d'être admis par tous.

\section{Intégration et altérité}

L'enseignement de l'espagnol reste un objectif prioritaire de l'association, une enquête que celle-ci a diligentée permit de mettre en lumière le sous-emploi de nombre d'Haïtiens, possédant par ailleurs certaines qualifications professionnelles, du seul fait de leur ignorance de cette langue. Moyennant une participation financière modique (de l'ordre de 2000 Bolívars soit environ 21 francs) destinée à l'achat de fournitures, un cours permanent a pu être mis en place. Par ailleurs les membres en cours d'alphabétisation sont entraînés à l'écoute des médias, ce qui permet un apprentissage plus rapide et une meilleure adaptation à la société d'accueil.

L'intégration visée n'est cependant pas à sens unique et c'est pourquoi est né le projet d'un journal bilingue (espagnol/créole) qui devrait bientôt voir le jour et être diffusé également hors du Venezuela afin de renforcer la visibilité de la communauté.

Le risque d'oubli de ses racines par la seconde génération est également au centre des préoccupations de l'association. La stratégie choisie passe par l'enseignement et plus précisément par la création d'une école maternelle où les enfants recevraient une formation bilingue intégrant les apports des deux cultures. Les réformes introduites récemment dans le système scolaire et notamment le projet « plantel» devraient favoriser la concrétisation de cette initiative.

Comme son nom l'indique la connaissance et le rayonnement de la culture haïtienne au Venezuela demeure l'objectif fondamental de l'association. Il s'agit là d'une tâche particulièrement difficile car 
celle-ci est dramatiquement absente de la scène artistique y compris dans le domaine musical qui est généralement plus favorable aux communautés noires. L'existence du groupe «combo de Haïti au Venezuela » dans les années 80 a été éphémère et n'a pas eu de postérité. Des ateliers de percussion et de théâtre sont en cours de réalisation en coopération avec des associations culturelles afrovénézuéliennes.

\section{Le lien afro-américain : un facteur d'intégration?}

Depuis une dizaine d'années on observe au Venezuela un renouveau de la culture noire, fruit du travail associatif ${ }^{32}$ mais qui s'était jusque-là, essentiellement manifesté au sein des communautés afro-vénézuéliennes. Peu à peu cependant, des échanges limités se sont mis en place entre ces dernières et des populations de la Caraïbe insulaire : Trinidad (Péninsule de Paria), Curaçao (Etats de Carabobo et Yaracuy). Il n'y avait pas eu d'intégration au Venezuela entre les noirs autochtones et immigrés dans les régions où ils avaient été amenés à se côtoyer: zones d'exploitation pétrolière (Lac de Maracaïbo) ou minière, grandes villes. Les causes de ce rejet mutuel ont été nombreuses : obstacle de la langue, concurrence sur le marché du travail, religions différentes.

Nous avons déjà évoqué les préjugés racistes dont sont victimes les Haïtiens, il convient de souligner que ceux-ci sont souvent partagés par la population de couleur elle-même qui a intériorisé ces schémas ${ }^{33}$. La société haïtienne n'est pas non plus exempte de ce type de manifestations qui attise le conflit socio-économico-politique entre noirs et mulâtres si bien instrumentalisé par le duvaliérisme ${ }^{34}$ et dont

32. Charier A., La culture afro-vénézuélienne déclin et renouveau: le cas du Barlovento, Villeneuve d'Ascq, Presses universitaires du septentrion, 1997.

33. Mijares M. M., Racismo y endoracismo en Barlovento, Fundacion Afroamérica, Caracas, 1997.

34. Nicholls. D., From Dessalines to Duvalier. Race, colour and national independence in Haïti, Cambridge, Cambridge University Press, 1979. 
des événements survenus ces derniers temps ont mis en évidence la permanence.

Le soutien apporté d'emblée par la fondation «Afroamérica » à l'association haïtienne n'en prend donc que plus de relief, surtout lorsque l'on sait qu'il s'agit de l'organisation culturelle noire la plus connue du pays. Cette aide a pris diverses formes mais elle a surtout été déterminante lorsqu'il fallut établir un contact avec le CONAC (Secrétariat d'Etat à la culture). Cet organisme envisagerait désormais de prendre en compte dans ses programmes d'attribution de subventions cette association représentante d'une communauté immigrée de la Caraïbe, ce qui serait en soi une première. Parallèlement ce rapprochement a permis à des membres des deux communautés de se rencontrer et de se connaître comme cela a été le cas dans le Barlovento; ces contacts ont entraîné une prise de conscience du fonds culturel commun qui unissait les deux populations au-delà des différences nées des hasards de l'histoire.

On a évoqué depuis longtemps et avec raison l'invisibilité sociale du Noir en Amérique Latine et l'ignorance réciproque qui existait entre ce continent et la Caraïbe insulaire non-hispanophone ${ }^{35}$; on a également opposé un mouvement pan-amérindien en plein essor à un mouvement pan-afro-américain inexistant. L'expérience décrite ici est peut-être un signe annonciateur d'une profonde modification à terme de cette situation.

\section{Perspectives}

L'organisation sur des bases participatives d'une communauté immigrée peut atténuer le choc du déracinement (la faire passer, pour reprendre les termes d'E. Ollivier, de la condition de population exilée à celle de population migrante) et l'association ainsi crée servir d'institution médiatrice avec la société d'accueil pour peu qu'elle

35. Serbin A., Bryan A., Vecinos indiferentes? El Caribe de habla inglesa y América Latina, Caracas, Ed. Nueva Sociedad, 1990. 
sache y trouver des partenaires (notamment sur la base d'une proximité socio-culturelle quand cela est possible). Au-delà de l'aventure particulière des Haïtiens du Venezuela et de la dynamique de recomposition identitaire qui semble désormais l'animer et qu'illustre, en autres, l'expérience de la «Maison de la culture haïtienne au Venezuela », au-delà du contexte spécifique du monde caribéen, apparaît la nécessité de la remise en cause des stratégies d'intégration en œuvre ici et là et qui aboutissent trop souvent à la marginalisation et à l'exclusion de populations qui ne demandent qu'à participer à l'action commune, à la condition que la valeur de leur contribution à un nouveau projet national soit reconnue et acceptée. A l'heure où les identités se construisent et se recontruisent au gré des mobilités, souvent dans des situations de multiculturalisme liées aux espaces urbains tant dans les pays du Sud que dans les nations développées, il est urgent de prendre en compte ces apports dans le cadre des choix politiques, sociaux et culturels ${ }^{36}$.

36. Sur cette problématique nous renvoyons notamment aux ouvrages suivants: Balibar E., Wallerstein I., Race, classe, nation. Les identités ambigües, Paris, La Découverte, 1989 ; Bissoondath N., Selling illusions. The cult of Multiculturalism in Canada, Toronto, Penguin Books, 1994 ; Lassalle D., Les minorités ethniques en Grande-Bretagne, Paris, L'Harmattan, 1997 ; Naïr S, Lucas J. (de), Le déplacement du monde, Paris, Ed. Kimé, 1996; Noiriel G., Le creuset français. Histoire de l'immigration $X I X-X X^{e}$ siècle, Paris, Seuil, 1988; Simon-Barouh I. (Coord.), Dynamique migratoire et rencontre ethnique, Paris, L'Harmattan, 1998 ; Torres-Saillat S., Hernandez R., The Dominican Americans, Greenwood Press, 1996 ; Weber A., L'émigration réunionnaise en France, Paris, L'Harmattan, 1994 ;Wieviorka M. (Coord.), Une société fragmentée ? Le multiculturalisme en débat, Paris, La Découverte, 1996. 


\section{Résumé}

L'immigration haïtienne au Venezuela a été encore peu étudiée, les motivations qui l'animent et les voies d'insertion dans la société d'accueil qu'elle emploie demeurent mal connues. Elle est cependant un exemple significatif de la complexité des problèmes liés aux processus migratoires et aux stratégies contradictoires d'assimilation/altérité qu'ils peuvent faire naître. L'expérience de la «Maison de la culture d'Haïti au Venezuela » ouvre des perspectives sur de nouvelles dynamiques de recomposition identitaire s'appuyant sur des solidarités ethno-culturelles émergentes.

\begin{abstract}
Haitian immigration in Venezuela has been subject to little examination to date; its driving motivations and means of integration into the host society remain poorly known. It is, however, a significant example of the complexity of the problems linked to the process of migration and contradictory strategies of assimilation/alterity they could cause. The experiences of the Haitian cultural center in Venezuela open perspectives on the new efforts of identity recomposition based on emerging ethno-cultural solidarity.
\end{abstract}

Mots-clés

Afro-américanisme - Haïti - Identité -

Problèmes migratoires - Racisme - Raimond Julien - Saint-Domingue - Venezuela 